We conclude that for the same degree of lung function impairment females tend to report more (severe) dyspnoea and cough, but less phlegm. Knowledge of this difference in reporting symptoms is important as symptoms often are the first step to a diagnosis of underlying airway disease.

0 @ERSpublications

For the same degree of lung function impairment females tend to report more (severe) dyspnoea and cough, but less phlegm http://ow.ly/mp2CF

Bernd Lamprecht ${ }^{1,2,12}$, Lowie E. Vanfleteren ${ }^{3,4,12}$, Michael Studnicka ${ }^{1}$, Michael Allison ${ }^{5}$, Mary Ann McBurnie ${ }^{5}$, William M. Vollmer ${ }^{5}$, Wan Cheng Tan $^{6}$, Rune Nielsen ${ }^{7}$, Pawel Nastalek ${ }^{8}$, Louisa Gnatiuc ${ }^{9}$, Bernhard Kaiser ${ }^{1}$,

Christer Janson ${ }^{10}$, Emiel F.M. Wouters ${ }^{3,4}$, Peter Burney ${ }^{9}$ and A. Sonia Buist ${ }^{11}$ for the BOLD collaborative research group ${ }^{1}$ Dept of Pulmonary Medicine, Paracelsus Medical University, Salzburg, ${ }^{2}$ Department of Pulmonary Medicine, General Hospital Linz (AKH), Linz, Austria. ${ }^{3}$ Dept of Respiratory Medicine, Maastricht University Medical Centre, Maastricht, ${ }^{4}$ Program Development, CIRO+, Centre of Expertise in Chronic Organ Failure, Horn, The Netherlands. ${ }^{5}$ Kaiser Permanente Center for Health Research, Portland, OR, and ${ }^{11}$ Oregon Health and Science University, Portland, OR, USA. ${ }^{6}$ UBC iCapture Centre for CardioPulmonary Research, St Paul's Hospital, Vancouver, BC, Canada. ${ }^{7}$ Dept of Thoracic Medicine, Haukeland University Hospital, Bergen, Norway. ${ }^{8}$ Dept of Medicine, Jagiellonian University School of Medicine, Krakow, Poland. ${ }^{9}$ Respiratory Epidemiology and Public Health, Imperial College, London, UK. ${ }^{10}$ Dept of Medical Sciences, Respiratory Medicine and Allergology, Uppsala University Uppsala, Sweden. ${ }^{12}$ Both authors contributed equally.

Correspondence: B. Lamprecht, Dept of Pulmonary Medicine, Paracelsus Medical University, Salzburg, Austria. E-mail: dr.lamprecht@a1.net

Received: March 172013 | Accepted: March 282013

Support statement: B. Lamprecht is the recipient of European Respiratory Society Short-term Research training Fellowship 326-2011.

Conflict of interest: Disclosures can be found alongside the online version of this article at www.erj.ersjournals.com

\title{
References
}

Ofir D, McBride I, Webb KA, et al. Gender differences in exertional dyspnea with advancing age [abstract]. Proc Am Thorac Soc 2006; 3: A224.

2 Buist AS, McBurnie MA, Vollmer WM, et al. International variation in the prevalence of COPD (the BOLD study): a population-based prevalence study. Lancet 2007; 370: 741-750.

3 Hankinson JL, Odencrantz JR, Fedan KB. Spirometric reference values from a sample of the general US population. Am J Respir Crit Care Med 1999; 159: 179-187.

4 Watson L, Vestbo J, Postma DS, et al. Gender differences in the management and experience of chronic obstuctive pulmonary disease. Respir Med 2004; 98: 1207-1213.

5 de Torres JP, Casanova C, Hernández C, et al. Gender and COPD in patients attending a pulmonary clinic. Chest 2005; 128: 2012-2016.

6 Black LF, Hyatt RE. Maximal respiratory pressures: normal values and relationship to age and sex. Am Rev Respir Dis 1969; 99: 696-702.

7 Camp PG, O’Donnell DE, Postma DS. Chronic obstructive pulmonary disease in men and women: myths and reality. Proc Am Thorac Soc 2009; 6: 535-538.

$8 \quad$ Becklake MR, Kauffmann F. Gender differences in airway behaviour over the human life span. Thorax 1999; 54: $1119-1138$.

9 Johnson JL, Campbell AC, Bowers M, et al. Understanding the social consequences of chronic obstructive pulmonary disease: the effects of stigma and gender. Proc Am Thorac Soc 2007; 4: 680-682.

\section{Do we need bacteriological confirmation of cure in uncomplicated tuberculosis?}

\author{
To the Editor:
}

Using data from English tuberculosis (TB) services, we investigate whether European guidelines recommending high levels of culture confirmation of pulmonary tuberculosis (PTB) cases and proof of bacteriological cure are achievable or needed.

The European Centre for Disease Prevention and Control (ECDC) recommends that $80 \%$ of pulmonary cases should be culture-confirmed [1]. In England, the Chief Medical Officer's action plan for TB [2], aims 
for $65 \%$ of PTB cases to be confirmed by laboratory culture. Currently, although the UK target has been met, the ECDC goal has yet to be achieved in the UK [3]. In $201170 \%$ of notified cases in the UK were culture-confirmed. As part of treatment outcome monitoring the international guidelines in Europe, including the Wolfheze and International Standards for TB Care documents $[4,5]$, recommend that clinicians report evidence of bacteriological cure, defined as documented conversion (to culture-negative) during the continuation phase [6], but monthly sputum smear and culture samples are recommended when treating multidrug resistant (MDR)-TB [7]. In the UK, despite the existence of national treatment outcome monitoring programme for 9 years, data on bacteriological cure are frequently not reported. We investigated: 1) why not all cases of notified PTB have microbiological confirmation, and from this suggest methods to improve rates of microbiological confirmation; and 2) the feasibility of obtaining objective evidence for bacteriological cure among culture-confirmed cases. Three respiratory centres in the UK were chosen from metropolitan areas (two in London and one in Bristol). These have large TB case loads and generate adequate sample sizes for analysis. Furthermore, the London TB register and records in Bristol made the data particularly suitable and accurate for retrospective analysis.

A retrospective case note and pathology dataset review was performed, and a bespoke data collection tool completed. This tool collected information about: demographic data (table 1); the site of TB disease; sputum smear and culture status at the start and end of treatment; whether the sample obtained at the start and end of treatment was from spontaneously expectorated sputum, induced sputum or via bronchoscopy; if sputum smear status and/or mycobacterial cultures were not done, not known, or if data were missing whether reasons for this were documented in the case notes; drug sensitivity patterns; and relapse rates 2 years after the study.

TABLE 1 Demographics, acid-fast bacilli smear and mycobacterial culture results at diagnosis of incident pulmonary tuberculosis cases, 2009

\begin{tabular}{|c|c|}
\hline \multicolumn{2}{|l|}{ Sex } \\
\hline Female & 52 \\
\hline Male & 71 \\
\hline Age years & $40 \pm 16$ \\
\hline \multicolumn{2}{|l|}{ Ethnic group } \\
\hline Black & 43 (35) \\
\hline ISC & $34(28)$ \\
\hline White & $28(23)$ \\
\hline Other & $18(14)$ \\
\hline \multicolumn{2}{|l|}{ Previous TB diagnosis } \\
\hline Yes & $8(6)$ \\
\hline No & $103(84)$ \\
\hline Missing data & $12(10)$ \\
\hline \multicolumn{2}{|l|}{ HIV status } \\
\hline Positive & $10(8)$ \\
\hline Negative & 97 (79) \\
\hline Missing data & $16(13)$ \\
\hline \multicolumn{2}{|l|}{ UK born } \\
\hline Yes & $24(20)$ \\
\hline No & $95(77)$ \\
\hline Missing data & $4(3)$ \\
\hline Years in UK if non-UK born & $11 \pm 15$ \\
\hline Smear positive & $69(56)$ \\
\hline Smear negative & $49(40)$ \\
\hline Smear not performed ${ }^{\#}$ & $3(2)$ \\
\hline Smear data missing & $2(2)$ \\
\hline Culture positive & $98(80)$ \\
\hline Culture negative & 19 (15) \\
\hline Culture not performed $^{\#}$ & $4(3)$ \\
\hline Culture data missing ${ }^{\pi}$ & $2(2)$ \\
\hline
\end{tabular}

Data are presented as $n, \mathrm{n}(\%)$ or mean \pm SD. $\mathrm{n}=123$. Black: Black African, Black Caribbean, Black Other; ISC: Indian Subcontinent (Indian, Pakistani, Bangladeshi). " : Not performed = test not requested; " ${ }^{\text {" }}$ Data missing = test requested elsewhere, but unavailable at time of case note review. 
All new notified cases of pulmonary Mycobacterium tuberculosis in 2009 who were $\geqslant 18$ years at the time of diagnosis at three hospitals in England (Imperial College Healthcare NHS Trust London; Royal Free London NHS Foundation Trust; and University Hospitals Bristol NHS Foundation Trust) were included.

Across the three hospitals, there were 123 newly notified PTB cases in 2009 (table 1). Of these, 8\% were known to be HIV positive. 112 (91\%) PTB cases had sputum or lung fluid samples sent for acid-fast bacilli (AFB) smear and mycobacterial culture at diagnosis. 5\% had lung biopsy or lymph node samples similarly examined.

At diagnosis, the total proportion of sputum smear-positive cases was $56 \%$ across all three hospitals and 40\% were smear-negative (table 1). From all 123 TB cases, the majority were confirmed with sputum culture. When sputum culture was negative, bronchoscopy contributed to the detection of another 15 $(12 \%)$ of the cases. In total, $80 \%$ were culture positive at diagnosis. Culture data were unavailable in six cases $(5 \%)$. The reasons identified for no culture data being available were: two samples were obtained by surgical teams who did not send them to microbiology; one had absence of respiratory symptoms; one had an initial diagnosis made external to the site of treatment; and in two cases no cause was found.

At treatment completion, the majority did not undergo a repeat culture for $M$. tuberculosis ( $82 \%$ ), $15 \%$ had a negative culture result and $2 \%$ had unavailable data. The main reasons for no culture sample being sent at the end of treatment were the individual: 1) was clinically asymptomatic $(28 \%) ; 2)$ had documented radiological resolution $(20 \%)$; 3) defaulted from care $(9 \%)$, or 4) transferred out from the local TB service $(6 \%)$. Ten $(8 \%)$ of the 123 cases were on an extended treatment period, due to either drug resistance or treatment interruption. They did not have further culture results available at the time of the initial audit. 2 years after treatment completion for those treated in 2009, we found that only one case had relapsed, who was known to have been non-adherent with initial treatment and to have pulmonary and pleural disease.

This study sought to evaluate why not all notified cases of pulmonary tuberculosis have diagnostic microbiological confirmation. We found that the great majority $(96 \%)$ had documented evidence of sputum, lung fluid or tissue samples being sent for smear and culture during diagnostic evaluation. This meets ECDC recommendations and is above the current UK threshold. Therefore, we recommend that the UK Chief Medical Officer should review and revise the UK guidelines upwards to conform with the European recommendations. In the few cases that did not have samples sent at diagnosis, this was mainly because clinicians without specialist experience in $\mathrm{TB}$ were involved in the diagnostic procedure and appeared less likely to consider mycobacterial disease, and hence send relevant material.

It is of interest that, despite cases where our post hoc analysis suggested a high pretest likelihood of TB, surgically-obtained samples were not always sent for mycobacterial culture. Surgical pathology checklists, that have been used successfully elsewhere [8], may help here by acting as a prompt for clinicians to consider infections such as TB in certain populations who would be regarded as being at "at risk" of TB, such as Asians presenting with lymph node masses.

At diagnosis, 56\% of subjects had AFB smear-positive disease and $80 \%$ had culture-positive results. This exceeds the national target for the latter (of 65\%), and meets that recommended by the ECDC. We reviewed information from three large English metropolitan TB services (two in London and one in Bristol). We cannot, therefore, extrapolate our findings to all national TB care providers. However, the results suggest that in most cases samples for mycobacterial diagnosis can be obtained, and that achieving improved culture confirmation actually requires better microbiological techniques, in addition to sending surgical specimens for culture.

We also investigated the practicality of obtaining bacteriological confirmation of cure among treated pulmonary culture-positive cases. At treatment completion, four-fifths were not tested for cure. This was because they were in good health and invasive tests were felt to be inappropriate by their managing clinicians. Both national and local data indicate low rates of relapse post anti-TB treatment [9], suggesting that the majority of subjects are cured with therapy. We believe it is not indicated, in the UK, to pursue end of therapy samples for mycobacterial culture when a patient has successfully completed treatment and is well. In light of this, we recommend that each European country, based on their treatment completion and disease relapse rates plus local drug resistance prevalence, should review their policy and decide whether a final sputum culture is likely to be of value and cost-effective.

We conclude that microbiological diagnosis of PTB is performed appropriately. However, obtaining evidence of cure is impractical and unnecessary in the majority of treated individuals in our UK hospital settings. We recommend that the European guidelines are revised. The World Health Organization allows for the reporting of treatment completion (i.e. those who complete a treatment regimen and do not have a 
negative smear or culture sample sent in the last month of treatment) as a successful outcome. This could be emphasised in future guidelines, with the proviso that if there is no proof of bacteriological cure, greater efforts should be made to improve initial microbiological TB diagnosis. This would enable individualised patient-specific drug susceptibility results to inform regimen choice and ensure that treatment completion is a robust indicator of success. In light of the new information from this study, we would suggest similar studies are performed in different settings both in the UK and elsewhere. The information obtained may allow for better selection of patients to be tested at treatment completion.

0 @ERSpublications

In UK hospitals obtaining evidence of TB cure is impractical and unnecessary in the majority of treated individuals http://ow.ly/mxhQM

Abbey Ursula Leahy ${ }^{1}$, Marc Lipman ${ }^{2}$, Martin Hetzel ${ }^{1}$, Onn Min Kon ${ }^{3}$, Susan Hopkins ${ }^{2}$ and Ibrahim Abubakar ${ }^{4}$ ${ }^{1}$ University Hospitals Bristol NHS Foundation Trust, Bristol, ${ }^{2}$ Royal Free London NHS Foundation Trust and University College London, London, ${ }^{3}$ Imperial College Healthcare NHS Trust and National Heart and Lung Institute, London, and

${ }^{4}$ Tuberculosis Section, Health Protection Agency Colindale and University College London, London, UK.

Correspondence: I. Abubakar, Tuberculosis Section, Health Protection Agency Colindale and University College London, London, UK. E-mail: i.abubakar@ucl.ac.uk

Received: Nov 252012 | Accepted after revision: March 022012

Conflict of interest: None declared.

\section{References}

1 European Centre for Disease Prevention and Control. Progressing towards TB elimination. A follow-up to the Framework Action Plan to Fight Tuberculosis in the European Union. Stockholm, ECDC, 2010.

2 Department of Health. Stopping Tuberculosis in England: An action plan from the Chief Medical Officer. http://webarchive.nationalarchives.gov.uk/20130107105354/http://www.dh.gov.uk/prod_consum_dh/groups/dh_ digitalassets/@dh/@en/documents/digitalasset/dh_4100860.pdf Date last accessed: July 3, 2013. Date last updated: October 7, 2004.

3 Health Protection Agency. Tuberculosis in the UK: 2011 report. London, HPA, 2011.

4 Veen J, Migliori GB, Raviglione M, et al. Harmonisation of TB control in the WHO European region: the history of the Wolfheze Workshops. Eur Respir J 2011; 37: 950-959.

5 Migliori GB, Zellweger JP, Abubakar I, et al. European union standards for tuberculosis care. Eur Respir J 2012; 39: 807-819.

6 Veen J, Raviglione M, Rieder HL, et al. Standardized tuberculosis treatment outcome monitoring in Europe. Recommendations of a Working Group of the World Health Organization (WHO) and the European Region of the International Union Against Tuberculosis and Lung Disease (IUATLD) for uniform reporting by cohort analysis of treatment outcome in tuberculosis patients. Eur Respir J 1998; 12: 505-510.

7 Falzon D, Jaramillo E, Schünemann HJ, et al. WHO guidelines for the programmatic management of drug-resistant tuberculosis: 2011 update. Eur Respir J 2011; 38: 516-528.

8 Vats A, Vincent CA, Nagpal K, et al. Practical challenges of introducing WHO surgical checklist: UK pilot experience. BMJ 2010; 340: b5433.

9 Crofts JP, Andrews NJ, Barker RD, et al. Risk factors for recurrent tuberculosis in England and Wales, 1998-2005. Thorax 2010; 65: 310-314.

\section{Adherence to positive airway pressure in non-sleepy patients with obstructive sleep apnoea}

\section{To the Editor:}

Complaints of excessive daytime sleepiness (EDS) are absent in many individuals with obstructive sleep apnoea (OSA). The influence of EDS prior to treatment on continuous positive airway pressure (CPAP) adherence has not been clearly determined $[1,2]$. The aim of this prospective cohort study was to evaluate the adherence and perceived benefit during long-term CPAP therapy in a "real life" population of nonsleepy OSA patients. 